\title{
Absceso hepático: Serie de 107 casos y revisión de la literatura
}

Andrés Fantuzzi S., Nicolás Albertz A., Antonia Valenzuela V., Nivia Estuardo A. y Ariel Castro L.

\section{Hepatic abscess: Series of 107 cases and literature review}

Liver abscess is a rare disease with high morbidity and mortality. Objective and Method: Description of liver abscess cases attended in the Universidad de Chile Clinical Hospital between 2000 and 2007 and review of the literature on the topic. Results: For 107 cases reviewed, mean age was 59 years. Most common symptoms were fever and abdominal pain with an average duration of 19 days. Comorbidity: twenty five percent of patients had Diabetes Mellitus type 2, seven percent were immunosuppressed patients and 24\% had undergone invasive procedures. Not achieved identify the origin in most cases. The most requested image test was abdominal CT. In 49/75 (65.3\%) the abscess culture allowed to establish the etiology. Bacteremia was present in $17 / 68(25 \%)$ of cases. Most cases were treated with two or more antibiotics and percutaneous drainage. Conclusion: Clinical manifestations and resolution of liver abscess in this series are consistent with those described in the literature.

Key words: Liver abscess, infection, sepsis.

Palabras clave: Absceso hepático, infección, sepsis.

\section{Introducción}

$\mathrm{E}$ 1 absceso hepático es una patología infrecuente pero altamente letal ${ }^{1}$. Su incidencia varía entre $0,008 \%$ y $0,022 \%$ en pacientes hospitalizados $^{2,3}$, aumentando en el último tiempo por el manejo enérgico de patologías hepato-biliares y neoplasias pancreáticas ${ }^{1}$. Su mortalidad fluctúa entre 8 y $31 \% \%^{2,4}$, aunque está disminuyendo gracias a la introducción del drenaje percutáneo, procedimiento de bajo riesgo para los pacientes críticos y que constituye actualmente la técnica diagnóstica y terapéutica de elección ${ }^{2,5,6}$.

Es necesario un alto índice de sospecha clínica para realizar el diagnóstico precoz y permitir un oportuno drenaje percutáneo, la administración de antimicrobiano de espectro apropiado y el tratamiento enérgico de las complicaciones sistémicas concomitantes; estas medidas han aumentado la sobrevida y han mejorado el curso clínico de esta patología. La ultrasonografía y la TAC han facilitado el diagnóstico temprano y el drena$\mathrm{je}^{7}$. Así es como la TAC de abdomen se ha constituido en la técnica diagnóstica no invasora de elección.

Hay evidencia en la literatura médica de un cambio significativo, en las últimas cuatro décadas, de los patógenos involucrados en la etiología de los abscesos hepáticos ${ }^{1,2,7}$. Mientras, Escherichia coli ha disminuido progresivamente su incidencia, se ha observado un aumento de Klebsiella sp, Pseudomonas sp,
Streptococcus sp y Staphylococcus sp. Además, se ha descrito la emergencia de abscesos causados por cepas bacterianas resistentes a múltiples antimicrobianos, abscesos fúngicos y polimicrobianos como un fenómeno relativamente nuevo ${ }^{8}$.

La patología biliar benigna es aún una causal importante de absceso hepático; sin embargo, el factor determinante más frecuente en nuevas series es la obstrucción maligna de la vía biliar9. La diabetes mellitus es la patología concomitante más común y aumenta en forma significativa la mortalidad en esta patología 5 . Por último, algunos estudios han demostrado la asociación entre estados de inmunosupresión y absceso hepático. A pesar de lo anterior, el número de abscesos criptogénicos sigue siendo importante alcanzando en series comunicadas hasta $42,1 \%$ de los casos $^{5}$.

El objetivo de este trabajo es describir las características clínicas de los pacientes con diagnóstico de absceso hepático en nuestra institución y la revisión de la literatura científica respecto del tema.

\section{Pacientes y Método}

Se realizó un estudio descriptivo y retrospectivo. Para ello se revisaron los registros clínicos del Hospital Clínico de la Universidad de Chile desde enero del año 2000 hasta diciembre del año 2007. Criterios de inclusión: pacientes egresados con diagnóstico de
Hospital Clínico Universidad de Chile, Santiago, Chile Sección Medicina Interna

Recibido: 11 abril 2008 Aceptado: 20 noviembre 2008

Correspondencia a: Nicolás Albertz Arévalo medinter@redclinicauchile.cl, nicolas.albertz@gmail.com 
absceso hepático, dato facilitado por el departamento de estadísticas de nuestro hospital, y que además, al momento de revisar los antecedentes del paciente, se cumplieran al menos alguno de los criterios que se enumeran a continuación:

- Hallazgo de una o varias imágenes intra-hepáticas de características líquidas por medio de ecotomografía y/o TAC, asociada a la sospecha de absceso hepático basada en el cuadro clínico, exámenes de laboratorio y/o estudio microbiológico.

- Hallazgo de una o varias imágenes intra-hepáticas de características líquidas por medio de ecotomografía y/o TAC, con posterior drenaje de pus.

- Hallazgo de colección intra-hepática en relación a algún procedimiento quirúrgico.

Criterios de exclusión: aquellos pacientes informados inicialmente como absceso hepático que al revisar su ficha clínica no cumpliera con estos puntos, o bien correspondieran a otros diagnósticos.

Se recopilaron los siguientes datos: Características bio-demográficas, antecedentes de inmunosupresión, procedimientos diagnósticos y/o terapéuticos anteriores, motivo de consulta, foco séptico primario, estudios por imágenes realizados, parámetros inflamatorios de laboratorio, pruebas de función hepática, microorganismos aislados, ubicación del absceso, técnica de drenaje y estado al egreso hospitalario.

Se utilizaron medidas estadísticas de distribución y posición siendo procesadas con el programa STATA 8.0.

Tabla 1. Características bio-demográficas y de morbilidad en 107 pacientes con absceso hepático

\begin{tabular}{lrr} 
Población total & $\mathrm{n}=107$ \\
Edad (años) & $59 \pm 16(18-90)$ \\
Sexo & hombres $(51,4 \%)$ \\
Síntomas & $\mathrm{n}^{\circ}$ pacientes $(\%)$ \\
$\quad$ Fiebre & $66 \quad(61,7)$ \\
Dolor abdominal & 61 & $(57,0)$ \\
Ictericia & 21 & $(19,6)$ \\
Calofríos & 18 & $(16,8)$ \\
Duración de la sintomatología (días) & $\pm 25,5$ & $(0-180)$ \\
Hallazgos al examen físico & $\mathrm{n}^{\circ}$ pacientes $(\%)$ \\
$\quad$ Fiebre & 48 & $(44,9)$ \\
$\quad$ Sensibilidad abdominal & 74 & $(69,2)$ \\
Patología concomitante & \multicolumn{2}{c}{} \\
$\quad$ Diabetes mellitus 2 & 27 & $(25,2)$ \\
Inmunosupresión & 8 & $(7,5)$ \\
Procedimiento sobre la vía biliar & 26 & $(24,3)$ \\
\hline
\end{tabular}

\section{Resultados}

Datos bio-demográficos. Se identificaron 112 casos, de los cuales se analizaron 107. Los 5 restantes no cumplieron los criterios de inclusión. La edad promedio fue de 59 años (18-90 años). La distribución por sexo observada fue de 55 hombres $(51,4 \%)$ y 52 mujeres $(48,6 \%)$.

Co-morbilidad: de la población estudiada, 25\% padecía de diabetes mellitus tipo 2, 7\% estaba con terapia inmunosupresora y en $24 \%$ se encontró relación con algún procedimiento previo (Tabla 1).

Presentación clínica. Los síntomas y signos consignados como motivo de consulta fueron: fiebre (61\%) y dolor abdominal (57\%). Duración de la sintomatología hasta el diagnóstico: promedio de 19 días (0-180 días). Al momento del ingreso sólo en $44 \%$ de los pacientes se registró fiebre $>38^{\circ} \mathrm{C}$ y en $69 \%$ de los casos existía algún grado de sensibilidad abdominal.

Patología y factores asociados. En cuanto a los factores determinantes, en 57 pacientes $(53,2 \%)$ no se logró identificar su origen, catalogándose como idiopático. El antecedente de cirugía abdominal (no vía biliar) u otros se encontró en 23 casos $(21,5 \%)$, colangitis en $21(19,6 \%)$, infección vía hematógena $(2,8 \%)$ y diverticulitis $(2,8 \%)$ (Tabla 2$)$

Hallazgos de laboratorio. En 55 casos $(51,4 \%)$ se encontró sobre 12.000 leucocitos $/ \mathrm{mm}^{3}$ y en 76 pacientes $(71 \%)$ se obtuvo un nivel de PCR mayor de 100 mg/L (Tabla 3). Cabe consignar que, dos pacientes tuvieron serología positiva para Fasciola hepática (ELISA y fijación de complemento).

Estudio por imágenes. Se realizó en los 107 pacientes. La TAC de abdomen se llevó a cabo en 95 pacientes $(88,7 \%)$, ultrasonido se solicitó en 68 casos $(63,5 \%)$ y en 9 pacientes $(8,4 \%)$ se practicó $\mathrm{RM}$ de hígado (Tabla 3).

Estudios microbiológicos. Se tomaron hemocultivos

\begin{tabular}{|c|c|c|}
\hline Patología/condición & $n^{\circ}$ casos & $(\%)$ \\
\hline Colangitis & 21 & $(19,6)$ \\
\hline Diverticulitis & 3 & $(2,8)$ \\
\hline Hematógena & 3 & $(2,8)$ \\
\hline Idiopática & 57 & $(53,3)$ \\
\hline Otras & 23 & $(21,5)$ \\
\hline \multicolumn{3}{|c|}{$\begin{array}{l}\text { Otras incluye: Cirugías intrabdominales no Gastrectomía - } \\
\text { Proceso inflamatorio Hepatocarcinomade vía biliar - Quiste } \\
\text { hidatídico hepático-pelviano - Cáncer de páncreas- }\end{array}$} \\
\hline
\end{tabular}


en 68 pacientes $(63,5 \%)$, constatándose bacteriemia en 17 casos (25\%). El agente más aislado fue Staphylococcus aureus MR (n: 6 casos, 35,2\%) (Tabla 4).

Los cultivos de secreción obtenida por punción y/o en forma intra-operatoria de absceso hepático se tomaron en 75 pacientes (70\%). De estos resultaron 49 positivos $(65,3 \%)$, siendo monomicrobianos: $40(81,6 \%)$ y polimicrobianos: $9(18,3 \%)$. Se aislaron anaerobios en 13 muestras $(26,5 \%)$, enterobacteriaceas en 17 $(34,7 \%)$, bacilos gramnegativos no fermentadores en 5 $(10,2 \%)$ y cocáceas grampositivas en 21 muestras (42,8\%). (Tabla 5).

Antibioterapia y drenaje. Como esquema antimicrobiano inicial, en 82 pacientes $(78,8 \%)$ se administraron dos fármacos, en 15 casos $(14,4 \%)$ uno y en 7 pacientes $(6,7 \%)$ se usaron tres antimicrobianos. En 89 pacientes se utilizó más de un fármaco $(85,6 \%)$ y el tiempo de latencia hasta el inicio de la antibioterapia fue, en promedio, de 1,63 días ( 0 - 17 días). Las combinaciones más usadas fueron: cefalosporina de $3^{\text {a }}$ generación más anti-anaeróbico en 58 pacientes $(55,7 \%)$ y quinolona más anti-anaeróbico en 13 casos $(14,4 \%)$. Como monoterapia inicial se indicó quinolona en 6 pacientes $(5,7 \%)$ y cefalosporina de $3^{\text {a }}$ generación en 4 pacientes $(3,8 \%)$. Un paciente recibió terapia de tercera línea con carbapenem más metronidazol al ingreso y en 7 casos $(6,7 \%)$ se inició terapia tri-asociada con cefalosporina de $3^{\text {a }}$ generación, anti-anaeróbico y ampicilina.

Como terapia anti-anaeróbica se utilizó metronidazol en 82 casos $(78,8 \%)$ y clindamicina en 5 casos $(4,8 \%)$ (Tabla 6).

El tratamiento no farmacológico consistió en drenaje percutáneo en 79 pacientes $(73,8 \%)$. En 18 casos $(16,8 \%)$ se requirió terapia por radiología intervencional más cirugía clásica.

El $87,8 \%$ de los pacientes fue sometido a más de un tratamiento y sólo 13 pacientes $(12,1 \%)$ recibieron terapia anti-infecciosa exclusiva (Tabla 7).

Complicaciones y pronóstico. Se produjeron seis muertes (letalidad 5,6\%). Cinco de estos pacientes tenían más de 65 años (65-80 años). La causa de muerte consignada fue shock séptico en todos los casos, asociándose en uno de ellos a procedimiento de la vía biliar (colangiopancreatografía retrógrada endoscópica- ERCP en su sigla inglesa), otro asociado a cirugía bilio-digestiva, dos casos a post-cirugía intra-abdominal de otro origen y un caso a inmunosupresión. En otro paciente no se encontró asociación con procedimiento ni se estableció que tuviera co-morbilidad, completando las seis defunciones enunciadas.

\begin{tabular}{|c|c|c|}
\hline \multirow{2}{*}{$\begin{array}{l}\text { Exámenes de laboratorio } \\
\text { Leucocitos } / \mathrm{mm}^{3}\end{array}$} & \multicolumn{2}{|c|}{$\begin{array}{l}\text { (promedio, desv, } \min / \text { máx), } \\
\% \text {, mediana, percentiles }(25 \% \text { / } 75 \%)\end{array}$} \\
\hline & 13,967 & $(0-48.900)$ \\
\hline PCR mg/L & $171 \pm 119$ & $(0-425)$ \\
\hline $\mathrm{LDH} \mathrm{U} / \mathrm{L}$ & $542 \pm 1118$ & $(0-10.888)$ \\
\hline Albuminemia $\mathrm{gr} / \mathrm{dl}$ & $2,34 \pm 1,25$ & $(0-5)$ \\
\hline Protrombinemia & $70 \%$ & $(60-83 \%)$ \\
\hline VHS $\mathrm{mm} / \mathrm{h}$ & $76 \pm 35$ & $(0-129)$ \\
\hline Bilirrubinemia total mg/dl & $1,88 \pm 2,58$ & $(0-14,9)$ \\
\hline Bilirrubinemia directa $\mathrm{mg} / \mathrm{dl}$ & $1,63 \pm 4,54$ & $(0-42)$ \\
\hline GOT U/L & $124 \pm 348$ & $(0-3.290)$ \\
\hline GGT U/L & $246 \pm 254$ & $(0-1.355)$ \\
\hline Fosfatasas alcalinas U/L & $265 \pm 207$ & $(0-1.300)$ \\
\hline Exámenes imagenológicos & $n^{\circ}$ pacientes & D $\quad(\%)$ \\
\hline Ecografía & 68 & $(63,6)$ \\
\hline Tomografía axial computada & 95 & $(88,8)$ \\
\hline Resonancia magnética & 9 & $(8,4)$ \\
\hline \multicolumn{3}{|c|}{$\begin{array}{l}\text { PCR: Proteína C reactiva, LDH: Deshidrogenasa láctica, GOT: Aspartato aminotransferasa, } \\
\text { GGT: Gammaglutamiltranspeptidasa, VHS: Velocidad de sedimentación globular }\end{array}$} \\
\hline
\end{tabular}

\begin{tabular}{|c|c|c|}
\hline & exámenes & (\%) \\
\hline Hemocultivos & 68 & $(63,5)$ \\
\hline Hemocultivos positivos & 17 & $(25,0)$ \\
\hline \multicolumn{3}{|l|}{ Microorganismo aislado } \\
\hline Staphylococcus aureus & 6 & $(35,3)$ \\
\hline Pseudomonas aeruginosa & 2 & $(11,7)$ \\
\hline Klebsiella pneumoniae & 2 & $(11,7)$ \\
\hline Staphylococcus coagulasa negativa & 2 & $(11,7)$ \\
\hline Enterococcus sp & 1 & $(5,9)$ \\
\hline Citrobacter freundii & 1 & $(5,9)$ \\
\hline Klebsiella oxytoca & 1 & $(5,9)$ \\
\hline Streptococcus agalactiae & 1 & $(5,9)$ \\
\hline Streptococcus grupo viridans & 1 & $(5,9)$ \\
\hline Escherichia coli & 1 & $(5,9)$ \\
\hline
\end{tabular}


Tabla 5. Hallazgos microbiológicos en pus obtenido de 107 pacientes con abscesos hepáticos

\begin{tabular}{|lcl|}
\hline & $\mathbf{n}^{\circ}$ exámenes & $\mathbf{( \% )}$ \\
Cultivos de pus & 75 & $(70,0)$ \\
Cultivos de pus & 49 & $(65,3)$ \\
Microorganismos aislados & & \\
\hline Klebsiella pneumonia & 10 & $(20,4)$ \\
Escherichia coli & 7 & $(14,3)$ \\
\hline Pseudomonas aeruginosa & 5 & $(10,2)$ \\
\hline Fusobacterium sp & 5 & $(10,2)$ \\
\hline Enterococcus sp & 5 & $(10,2)$ \\
Streptococcus sp & 4 & $(8,2)$ \\
\hline Staphylococcus aureus & 4 & $(8,2)$ \\
Proteus mirabilis & 3 & $(6,1)$ \\
\hline Candida sp & 3 & $(6,1)$ \\
Bacteroides sp & 3 & $(6,1)$ \\
\hline BGN sin tipificación & 2 & $(4,1)$ \\
Morganella morganii & 2 & $(4,1)$ \\
\hline Peptostreptococcus & 2 & $(4,1)$ \\
Clostridium perfringens & 2 & $(4,1)$ \\
\hline Cocáceas G(+) sin tipificación & 2 & $(4,1)$ \\
\hline Streptococcus grupo viridans & 2 & $(4,1)$ \\
\hline Streptococcus constellatus & 1 & $(2,0)$ \\
\hline Staphylococcus coagulasa negativa & 1 & $(2,0)$ \\
\hline Veillonella sp & 1 & $(2,0)$ \\
\hline BGN: bacilo gramnegativo & & \\
\hline
\end{tabular}

Tabla 7. Estrategia terapéutica aplicada en 107 pacientes con abscesos hepáticos

\begin{tabular}{|lcc|}
\hline Tipo de intervención terapéutica & n pacientes & $(\%)$ \\
\hline Rx intervencional + antimicrobianos & 60 & $(56,1)$ \\
Cirugía + antimicrobianos & 13 & $(12,1)$ \\
No invasora (sólo antimicrobianos) & 13 & $(12,1)$ \\
Rx intervencional + cirugía + antimicrobianos & 18 & $(1,8)$ \\
\hline Sólo cirugía & 2 & $(0,9)$ \\
Sólo radiología intervencional & 1 & $(73,8)$ \\
\hline Total de pacientes & 79 & $(87,8)$ \\
Radiología intervencional & 94 & \\
\hline Más de un tratamiento & & \\
\hline Rx: Radiología & & \\
\hline
\end{tabular}

\section{Discusión}

El absceso hepático sigue siendo una patología con alta letalidad ${ }^{1,10}$. Los avances en las últimas décadas en nuevos métodos diagnósticos y terapéuticos han tenido impacto en las características epidemio-
Tabla 6. Tratamiento antimicrobiano empleado en 107 pacientes con abscesos hepáticos

\begin{tabular}{lcc} 
Fármaco utilizado & n pacientes & $(\%)$ \\
Metronidazol & 82 & $(77,9)$ \\
Ceftriaxona & 76 & $(73,1)$ \\
Ciprofloxacina & 18 & $(17,3)$ \\
Clindamicina & 5 & $(4,8)$ \\
Cefoperazona/sulbactam & 2 & $(1,9)$ \\
Cefazolina & 2 & $(1,9)$ \\
Levofloxacina & 2 & $(1,9)$ \\
Ampicilina & 2 & $(1,9)$ \\
Imipenem & 1 & $(1,0)$ \\
Amikacina & 1 & $(1,0)$ \\
Claritromicina & 1 & $(1,0)$ \\
Cloxacilina & 1 & $(1,0)$ \\
Anti-anaeróbicos & & \\
(Metronidazol-clindamicina) & 87 & $(83,6)$ \\
Cefalosporinas & 80 & $(76,9)$ \\
Fluoroquinolonas & 20 & $(20,2)$ \\
Otros $\beta$-lactámicos & 3 & $(2,9)$ \\
Otros & 2 & $(1,9)$ \\
Carbapenémicos & 1 & $(1,0)$ \\
& & \\
\hline
\end{tabular}

lógicas y en el tratamiento de los pacientes, situación que nos motivó a realizar este estudio.

El principal origen de absceso hepático en nuestra serie fue criptogénico en $53,3 \%$ de los casos. La patología biliar, fundamentalmente colangitis, sigue constituyendo un factor determinante de importancia, hecho reiteradamente descrito en la literatura médica ${ }^{2,5,8}$. El cambio descrito por Huang y cols ${ }^{1,}$ con respecto al aumento de la patología obstructiva maligna de la vía biliar como principal causa de absceso hepático, en parte debido al manejo más enérgico de estos pacientes, no se confirmó con nuestros datos.

Co-morbilidad. El 25,2\% de los pacientes tenía antecedentes de diabetes mellitus tipo 2 y $24,2 \%$ habían sido sometidos previamente a instrumentación de la vía biliar. Algunos estudios han mostrado además la importancia de la inmunosupresión ${ }^{3,8}$, en nuestros casos, estuvo asociada al diagnóstico de absceso hepático en $7,5 \%$ de los casos.

Concordante con lo publicado por muchos investigadores internacionales durante la década pasada ${ }^{1,2,7-9}$ e incluso recientemente en nuestro país ${ }^{11}$, Klebsiella pneumoniae fue el microorganismo aislado con mayor frecuencia en los cultivos de la lesión, con una alta prevalencia también en los hemocultivos, aunque el 
principal agente bacteriémico fue $S$. aureus. Se ha descrito en la literatura científica un aumento en la incidencia de abscesos hepáticos causados por Pseudomnas aeruginosa $a^{1,2}$, hallazgo que en nuestro estudio alcanzó casi a $10 \%$, tanto en cultivos de pus como en sangre. Es destacable además, la elevada frecuencia de Enterococcus sp $(10,2 \%)$ en los cultivos de secreción, atribuible probablemente a su prevalencia en la microbiota intrahospitalaria institucional ${ }^{12}$. Tanto el porcentaje de cultivos de pus como de hemocultivos negativos en nuestro estudio (en ambos casos cercano a $30 \%$ ) fue superior a lo reportado en la literatura que refiere $\sim 15 \%{ }^{2}$.

El desarrollo de la ecotomografía y de la TAC ha revolucionado el diagnóstico de muchas enfermeda$\operatorname{des}^{1,6}$. En este estudio se confirma la utilidad de estas técnicas imagenológicas para el diagnóstico de absceso hepático, habiendo sido utilizadas en esta serie en 63 y $88 \%$ de los pacientes, respectivamente. La RM fue utilizada sólo en $8 \%$ de los casos, cuando existía patología hepática subyacente. En esta serie la TAC fue la técnica radiológica más indicada para el diagnóstico de absceso hepático.

Recientemente, la introducción de técnicas de drenaje percutáneo ha modificado el tratamiento de los pacientes con absceso hepático ${ }^{6,11}$. En nuestro estudio, $73,8 \%$ de los pacientes fueron sometidos a terapia por radiología intervencional y $30 \%$ requirió tratamiento quirúrgico. Sólo $14 \%$ de los casos estudiados se sometió a cirugía clásica como único tratamiento no farmacológico. Muchos investigadores sostienen que una proporción significativa de pacientes pueden ser tratados con una combinación de tratamiento médico antimicrobiano y drenaje percutáneo guiado por imágenes, con excelentes resultados ${ }^{13,14}$. El presente estudio confirma que la introducción de estas nuevas técnicas de tratamiento menos invasoras, ampliamente utilizadas hoy en día, ha desplazado al manejo quirúrgico clásico.

Agradecimientos. A Jeannette Valdebenito M y Alberto Fica C.

\section{Resumen}

El absceso hepático es una patología infrecuente con alta morbimortalidad. Objetivo y método: Describir los casos de absceso hepático en el Hospital Clínico de la Universidad de Chile entre los años 2000 y 2007 y revisión de la literatura respecto del tema. Resultados: En 107 casos revisados, la edad promedio fue 59 años. Los síntomas más frecuentes fueron fiebre y dolor abdominal con una duración promedio de 19 días. El $25 \%$ de los casos presentaba como co-morbilidad diabetes mellitus tipo 2 , un $7 \%$ era inmunosuprimido y $24 \%$ había sido sometido a procedimiento invasor previo. No se logró identificar el origen en la mayoría de los casos. El estudio de imagen más solicitado fue la TC de abdomen. En 49/75 (65,3\%) se estableció la etiología mediante el cultivo del absceso. Se constató bacteriemia en 17/68 (25\%) pacientes. La mayoría de los casos se trataron con dos o más antimicrobianos y drenaje percutáneo. Conclusión: Las características de los casos de absceso hepático encontradas en este estudio concuerdan con lo descrito en la literatura médica.

\section{Referencias}

1.- Huang C, Pitt H A, Lipsett P, Osterman F, Lillemoe K, Cameron J, et al. Pyogenic hepatic abscess changing trends over 42 years. Ann Surg 1996; 223: 600-9.

2.- Lee K, Wong S, Sheen P. Pyogenic liver abscess: An audit of 10 years' experience and analysis of risk factors. Dig Surg 2001; 18: 459-66.

3.- Branum G D, Tyson G S, Branum M A, Meyers W C. Hepatic abscess changes in etiology, diagnosis and management. Am Surg 1990; 212: 655-62.

4.- Barakate M S, Stephen M S, Wangh R C, Gallahger P J, Solomon M J, Storey D W, et al. Pyogenic liver abscess: A review of 10 years' experience and management. Aust NZJ Surg 1999; 69: 205-9.

5.- Lee K T, Sheen P C, Chen J S, Ker C G. Pyogenic liver abscess: Multivariate analysis of risk factors. World J Surg 1991; 15:
372- 7.

6.- Khan R, Hamid S, Abid S, Jafri W, Abbas Z, Islam $M$, et al. Predictive factors for early aspiration in liver abscess. World $\mathrm{J}$ Gastroenterol 2008; 14: 2089-93.

7.- Yinnon A M, Hadas-Halpern I, Shapiro M, Hershko C. The changing clinical spectrum of liver abscess: the Jerusalem experience. Postgrad Med J 1994; 70: 436-9.

8.- Branum G D, Tyson G S, Branum M A, Meyers W C. Hepatic abscess: changes in etiology, diagnosis and management. Ann Surg 1990; 212: 655-62.

9.- Yeh T, Jan Y, Jeng L, Hwang T, Chao T, Chien R, et al. Pyogenic liver abscesses in patients with malignant diseases. A report of 52 cases treated in a single institution. Arch Surg 1998; 133: 242 - 5.

10.- Ruiz-Hernández J J, León-Mazorra M, Conde-Martel A, Marchena-Gómez J, Hemmersbach-Miller M, Betancor-León P. Pyogenic liver abscesses: mortality-related factors. Eur J Gastroenterol Hepatol 2007; 19: 853-8.

11.- Castro M, Eulufí A, Campos P, Eulufi S, Eulufi C. Drenaje percutáneo de abscesos intraabdominales (AIA) guiados por TAC. Rev Chilena Cirugía 2004; 56: 346-9.

12.- Fica A, Jemenao M, Bilbao P, Ruiz G, Sakurada A, Pérez de Arce E, et al. Emergencia de infecciones por Enterococcus sp resistente a vancomicina en un hospital universitario en Chile. Rev Chil Infect 2007; 24: 462-71.

13.- Chung Y F, Tan Y M, Lui H F, Tay K H, Lo R H, Kurup A, et al. Management of pyogenic liver abscesses - percutaneous or open drainage? Singapore Med J 2007; 48: 1158-65.

14. - Lambiase R E, Deyoe L, Cronan J J, Dorfman G S. Percutaneous drainage of 335 consecutive abscesses: Results of primary drainage with 1- year follow-up. Radiology 1992; 184: 167-79. 